\title{
Volterra Type Integral Equation by the Whittaker Cardinal Expansion
}

\author{
K. Maleknejad ${ }^{*}$ a and M. Alizadeh ${ }^{\mathrm{b}}$ \\ ${ }^{a}$ Department of Mathematics, Iran University of Science \& Technology, Narmak, Tehran 16846 13114, Iran \\ ${ }^{b}$ Faculty of Science, Islamic Azad University of Kerman, Kerman, Iran
}

\begin{abstract}
In this study, we use Whittaker cardinal expansion and collocation method to approximate the solution of Volterra integral equation of the second kind numerically which is arisen in many mathematical, industrial and medical problems. Then convergence of this method will be discussed in $\mathrm{L}_{\alpha}$ space for every positive $\alpha$. At the end of the paper, some numerical examples are presented to guarantee the accuracy of this method.
\end{abstract}

AMS Subject Classification: 45B05, 33C15, 65L60, 41A55.

Keywords: Volterra Integral equation, Whittaker cardinal expansion, Collocation method, Sinc function, Clenshaw-Curtis quadrature.

\section{INTRODUCTION}

Volterra type integral equation has been arisen in many mathematical and engineering field, so that solving this kind of problems are more efficient and useful in many research branches. Analytical solution of this kind of equation is not accessible in general form of equation and we can only get an exact solution only in special cases. But in industrial problems we have not spatial cases so that we try to solve this kind of equations numerically in general format. Many numerical schemes are employed to give an approximate solution with sufficient accuracy [1-5]. In this study we want to discuss on convergence of projection method with Sinc function then present a numerical solution to this type of equation.

Consider Volterra integral equation of the second kind

$f(s)=\int_{a}^{s} k(s, t) f(t) d t+g(s)-\infty<c \leq s \leq d<\infty$

where $k(s, t)$ and $g(s)$ are known functions, but $f(t)$ is an unknown function. In the operator form of this equation, we have

$(I-\mathrm{K}) f=g$

where $I$ is the identity operator and $\mathcal{K}$ is an integral operator defined by

$\mathcal{K}: X \rightarrow X$

$\mathcal{K} f=\int_{a}^{s} k(s, t) f(t) d t$

with $X$ is a normed space.

*Address correspondence to this author at the Department of Mathematics, Iran University of Science \& Technology, Narmak, Tehran 1684613114 , Iran; E-mail: maleknejad@iust.ac.ir

\section{Background}

Sinc function is defined on the whole real line by $\operatorname{sinc}(x)= \begin{cases}\frac{\sin (\pi x)}{\pi x}, & x \neq 0 \\ 1 & x=0 .\end{cases}$

Now, we define the sequence of Sinc basis function for positive $h$ by

$$
S(k, h)(x)=\frac{\sin (\pi(x-k h) / h)}{\pi(x-k h) / h}
$$

where $k$ is an integer.

Definition 1 Let $h>0$, and let $W\left(\frac{\pi}{h}\right)$ denote the family of functions $f$ that are analytic in $\mathrm{C}$ (set of all complex functions), such that

$\int|f(t)|^{2} d t<\infty$

and for all $z$ in $C$ (set of all complex numbers)

$|f(z)| \leq c e^{\frac{\pi|z|}{h}}$

with $c$ a positive constant.

Definition 2 Let $d>0$ and $D_{d}$ denote the domain

$D_{d}=\{\omega \in \mathrm{C}:|\operatorname{Im}(\omega)|<d\}$

Now for every positive $\alpha, \beta$ we define $\mathrm{L}_{\alpha, \beta}\left(D_{d}\right)$ is a space of functions $f$ that are analytic in $D_{d}$ such that for some $c>0$ and all $z \in D_{d}$ we have

$|f(z)| \leq c \frac{\left|e^{\alpha z}\right|}{\left(1+\left|e^{z}\right|\right)^{\alpha+\beta}}$. 
If $\alpha=\beta$, we have $\mathrm{L}_{\alpha, \alpha}\left(D_{d}\right)$, but for simplicity we denote $\mathrm{L}_{\alpha}\left(D_{d}\right)$ instead of $\mathrm{L}_{\alpha, \alpha}\left(D_{d}\right)$.

Theorem 1 Let $h>0$, the sequence $\left\{h^{-\frac{1}{2}} S(k, h)\right\}_{k=-\infty}^{\infty}$ is complete orthonormal sequence in $W\left(\frac{\pi}{h}\right)$. Every $f$ in $W\left(\frac{\pi}{h}\right)$ has the cardinal series representation

$f(x)=C(f, h)(x)=\sum_{k=-\infty}^{\infty} f(k h) S(k, h)(x)$

Proof. [6] page 91.

For $h>0$, the series $C(f, h)(x)$ is called the whittaker cardinal expansion of $f$ whenever this series converges [6].

\section{PROJECTION METHOD}

In projection methods such as collocation method for solving Eq.(2), we assume that $X_{n} \subset X=\mathrm{L}_{\alpha}$ and $\left\{\phi_{i}\right\} \quad i=1, \ldots, n$ are basis functions for $X_{n}$. By this assumption we try to solve integral equation of the second kind in a space with finite elements $[7,8]$.

In this paper, $X$ is $W\left(\frac{\pi}{h}\right)$ for every positive $h$ which is defined in next section and $X_{n}$ is a space constructed by Sinc basis functions. By using Whittaker cardinal expansion we can approximate every function in this space.

Now, we assume $X_{n}$ is a subspace of $W\left(\frac{\pi}{h}\right)$ constructed by orthonormal sequence $\left\{h^{-1 / 2} S(k, h)\right\}_{k=-N}^{N}$ [6]. Thus, we approximate an unknown function $f(s)$ by this orthonormal sequence, so by sampling theorem we have

$f(t) \approx C_{N}(f, h)(t)=\sum_{k=-N}^{N} f(k h) S(k, h)(t) \quad \forall h>0$

then by substituting this in integral equation we get

$$
\begin{aligned}
& \sum_{k=-N}^{N} f(k h) S(k, h)(s) \\
& =\int_{a}^{s} k(s, t) \sum_{k=-N}^{N} f(k h) S(k, h)(t) d t+g(s) .
\end{aligned}
$$

Now, we define residual equation by

$$
\begin{aligned}
& R_{N}(s)=\sum_{k=-N}^{N} f(k h) S(k, h)(s) \\
& -\int_{a}^{s} k(s, t) \sum_{k=-N}^{N} f(k h) S(k, h)(t) d t-g(s)
\end{aligned}
$$

for determining the unknown coefficients $f(k h)$ 's we select some collocation points such that

$R_{N}\left(s_{i}\right)=0 \quad i=0,1, \ldots, 2 N$

in this paper collocation points are $s_{i}=a+\frac{i(d-a)}{2 N} \quad i=0,1, \ldots, 2 N$

so that we have a system of linear equations $A_{N} X=b_{N}$ where

$$
\begin{aligned}
& A_{N}=\left[S(k, h)\left(s_{i}\right)-\int_{a}^{s_{i}} k\left(s_{i}, t\right) S(k, h)(t) d t\right]_{k=-N}^{N} \\
& b_{N}=\left[g\left(s_{i}\right)\right] \quad i=0,1, \ldots, 2 N \\
& X^{T}=[f(k h)]_{k=-N}^{N}
\end{aligned}
$$

In this system of linear equations we need to determine $\int_{a}^{s_{i}} k\left(s_{i}, t\right) S(k, h)(t) d t$. In this paper, we use a known numerical integration formula known as Clenshaw-Curtis method [9] to estimate this integral term and evaluate the elements of coefficient matrix $A$.

\section{MAIN RESULTS}

In this section, we want to investigate the convergence of this method for Volterra integral equation of the second kind by collocation method. To achieve this goal, firstly, we introduce definitions and theorems of Sinc approximation in strip [6], then use them to prove the convergence of our method.

Definition 3 Let $\lambda>0$ and $D_{\lambda}$ denote the domain

$D_{\lambda}=\{\omega \in \mathrm{C}:|\operatorname{Im}(\omega)|<\lambda\}$

Now for every positive $\alpha, \beta$ we define $\mathrm{L}_{\alpha, \beta}\left(D_{\lambda}\right)$ is a space of functions $f$ that are analytic in $D_{\lambda}$ such that for some $c>0$ and all $z \in D_{\lambda}$ we have

$|f(z)| \leq c \frac{\left|e^{\alpha z}\right|}{\left(1+\left|e^{z}\right|\right)^{\alpha+\beta}}$.

If $\alpha=\beta$ we have $\mathrm{L}_{\alpha, \alpha}\left(D_{\lambda}\right)$ but for simplicity we denote $\mathrm{L}_{\alpha}\left(D_{\lambda}\right)$ instead of $\mathrm{L}_{\alpha, \alpha}\left(D_{\lambda}\right)$.

Theorem 2 Let $f \in \mathrm{L}_{\alpha}\left(D_{\lambda}\right)$ for $\alpha>0$ and taking $h=\left(\frac{\pi \lambda}{\alpha N}\right)^{1 / 2}$ then there exists a positive number $c_{1}$, depending only on $f, \lambda, \alpha, y$ such that for $s=2$ or $s=\infty$

$$
\begin{aligned}
& \left\|E_{N}(f, h)(.+i y)\right\|_{s} \\
& =\left\|f(.+i y)-\sum_{k=-N}^{N} f(k h) S(k, h)(.+i y)\right\|_{s} \\
& \leq c_{1} N^{(1-1 / s) / 2} \exp \left\{-\left(\frac{\pi \alpha}{\lambda}\right)^{1 / 2}(\lambda-|y|) N^{1 / 2}\right\}
\end{aligned}
$$

Proof. [6] page 137.

In this paper we choose $s=\infty$ and for the real case we can substitute $y=0$ so that if $(\pi \alpha \lambda)^{1 / 2}=c_{2}$ then

$\left\|E_{N}(f, h)(.)\right\|_{\infty} \leq c_{1} N^{1 / 2} \exp \left\{-c_{2} N^{1 / 2}\right\}$. 
Theorem 3 By regard to Eq.(1) where $k(s, t)$ is continuous on square $[c, d] \times[a, d] \quad$ and $f(t), g(t) \in \mathrm{L}_{\alpha}([a, d])$ for $\alpha>0$, if we define

$P_{N}^{\text {num }}(f)(t)=\sum_{k=-N}^{N} f^{n u m}(k h) S(k, h)(t)$

as a Sinc approximation of $f(t)$ where $f^{\text {num }}(k h)$ is the solution of linear system of equations constructed by collocation method then

$\left\|f-P_{N}^{\text {num }}(f)\right\|_{\infty}$

$\leq c_{1} N^{1 / 2} \exp \left\{-c_{2} N^{1 / 2}\right\}\left\{1+c_{3}\left\|A_{N}^{-1}\right\| \frac{2}{\pi}(3+\log (N))\right\}$

Proof. First of all we define the following projection operator

$f(t) \approx P_{N}^{\text {num }}(f)(t)=\sum_{k=-N}^{N} f^{\text {num }}(k h) S(k, h)(t)$

In this projection operator $f^{n u m}(k h)$ is the solution of linear system of equations $A_{N} X=b_{N}$, so that $f^{n u m}(k h)$ contains truncation error and round off error. Another projection that we need in this theorem is

$P_{N}(f)(t)=\sum_{k=-N}^{N} f(k h) S(k, h)(t)$

where $f(k h)$ contains only truncation error. Practically, we solve

$P_{N}^{n u m}(f)(s)=\int_{a}^{s} k(s, t) P_{N}^{n u m}(f)(t) d t+g(s)$

to find $f^{n u m}(k h)$. So that we have

$\left[f^{n u m}(k h)\right]_{k=-N}^{N}=A_{N}^{-1}\left[g\left(s_{i}\right)\right] \quad i=0,1, \ldots, 2 N$

But if we substitute $P_{N}(f)(t)=\sum_{k=-N}^{N} f(k h) S(k, h)(t)$ then we define the new right hand side for Eq.(1) that is denoted by $\hat{g}$ and estimated as follow;

$P_{N}(f)(s)+f(s)-P_{N}(f)(s)$

$=\int_{a}^{s} k(s, t)\left[f(t)-P_{N}(f)(t)+P_{N}(f)(t)\right] d t+g(s)$

$P_{N}(f)(s)-\int_{a}^{s} k(s, t) P_{N}(f)(t) d t=g(s)-f(s)+$

$P_{N}(f)(s)+\int_{a}^{s} k(s, t)\left[f(t)-P_{N}(f)(t)\right] d t$

then

$\hat{g}(s)=g(s)-f(s)+P_{N}(f)(s)+$

$\int_{a}^{s} k(s, t)\left[f(t)-P_{N}(f)(t)\right] d t$

now we have

$[f(k h)]_{k=-N}^{N}=A_{N}^{-1}\left[\hat{g}\left(s_{i}\right)\right] \quad i=0,1, \ldots, 2 N$

so that $\sup _{k \in S_{N}}\left|f^{n u m}(k h)-f(k h)\right| \leq\left\|A_{N}^{-1}\right\| \sup _{i \in S_{N}}\left|g\left(s_{i}\right)-\hat{g}\left(s_{i}\right)\right|$

where $S_{N}$ is all integers belong to $[-N, N]$ and $\left\|A_{N}^{-1}\right\|$ is the maximum norm on columns or rows, so

$\sup _{i \in S_{N}}\left|g\left(s_{i}\right)-\hat{g}\left(s_{i}\right)\right| \leq \sup \mid P_{N}(f)\left(s_{i}\right)-f\left(s_{i}\right)+$ $i \in S_{N}$

$\int_{a}^{s} k\left(s_{i}, t\right)\left[f(t)-P_{N}(f)(t)\right] d t$ ।

$\leq \sup _{i \in S_{N}}\left|P_{N}(f)\left(s_{i}\right)-f\left(s_{i}\right)\right|$

$+\sup _{i \in S_{N}}\left|\int_{a}^{s} k\left(s_{i}, t\right)\left[f(t)-P_{N}(f)(t)\right] d t\right|$

$\leq c_{1} N^{1 / 2} \exp \left\{-c_{2} N^{1 / 2}\right\}$

$+\sup _{t \in[a, d]}\left|k\left(s_{i}, t\right)\right| \sup _{t \in[a, d]}\left|\left[f(t)-P_{N}(f)(t)\right] \| d-a\right|$

$\leq c_{1} N^{1 / 2} \exp \left\{-c_{2} N^{1 / 2}\right\}+M|d-a| c_{1} N^{1 / 2} \exp \left\{-c_{2} N^{1 / 2}\right\}$

where $M=\sup _{t \in[a, d]}\left|k\left(s_{i}, t\right)\right|$ and let $(1+M|d-a|)=c_{3}$ so $\sup _{i \in S_{N}}\left|g\left(s_{i}\right)-\hat{g}\left(s_{i}\right)\right| \leq c_{1} c_{3} N^{1 / 2} \exp \left\{-c_{2} N^{1 / 2}\right\}$

and

$\sup _{k \in S_{N}}\left|f^{\text {num }}(k h)-f(k h)\right| \leq\left\|A_{N}^{-1}\right\| c_{1} c_{3} N^{1 / 2} \exp \left\{-c_{2} N^{1 / 2}\right\}$.

Now we have

$$
\begin{aligned}
& \sup _{t \in[a, d]}\left|P_{N}(f)(t)-P_{N}^{\text {num }}(f)(t)\right| \\
& =\sup _{t \in[a, d]}\left|\sum_{k=-N}^{N}\left[f(k h)-f^{n u m}(k h)\right] S(k, h)(t)\right| \\
& \leq \sup _{k \in S_{N}}\left|f(k h)-f^{\text {num }}(k h)\right| \sup _{t \in[a, d]} \sum_{k=-N}^{N}|S(k, h)(t)| \\
& \leq\left\|A_{N}^{-1}\right\| c_{1} c_{3} N^{1 / 2} \exp \left\{-c_{2} N^{1 / 2}\right\}\left(\frac{2}{\pi}\{3+\log (N)\}\right)
\end{aligned}
$$

By regard to the [6]

$\sup _{t \in[a, d]} \sum_{k=-N}^{N}|S(k, h)(t)| \leq \frac{2}{\pi}\{3+\log (N)\}$

Finally, we have

$\left\|f-P_{N}^{\text {num }}(f)\right\|$

$\leq\left\|f-P_{N}(f)\right\|+\left\|P_{N}(f)-P_{N}^{\text {num }}(f)\right\|$

$\leq c_{1} N^{1 / 2} \exp \left\{-c_{2} N^{1 / 2}\right\}+$

$\left\|A_{N}^{-1}\right\| c_{1} c_{3} N^{1 / 2} \exp \left\{-c_{2} N^{1 / 2}\right\} \frac{2}{\pi}\{3+\log (N)\}$

$\leq c_{1} N^{1 / 2} \exp \left\{-c_{2} N^{1 / 2}\right\}\left\{1+c_{3}\left\|A_{N}^{-1}\right\| \frac{2}{\pi}(3+\log (N))\right\}$

and proof of this theorem is completed. 


\section{NUMERICAL EXAMPLES}

In this section, we use relations (3),(4),(5) to convert an integral equation to system of linear equations, for this result we choose $\alpha=1$ and $\lambda=\pi$ so that $h=\frac{\pi}{\sqrt{N}}$. In this procedure we use Clenshaw-Curtis method to estimate $\int_{a}^{s_{i}} k\left(s_{i}, t\right) S(k, h)(t) d t$ numerically. In these examples $E_{N}$ is defined by

$E_{N}=\max _{i}\left|f\left(t_{i}\right)-P_{N}^{\text {num }}(f)\left(t_{i}\right)\right| \quad i=0,1, \ldots, 2 N$

where $t_{i}$ 's are the collocation points.

In the following, we present some examples then numerical results are shown in Table $\mathbf{1}$, and these results are easily Comparable against other numerical methods which were appeared in [5-9] in points of convergence rate and error bound.

Example 1. In this example we solve Eq.(1) with

$k(s, t)=\frac{(s-t)^{2}}{1+t^{2}}$

$g(s)=\sqrt{1+s^{2}}-s\left(2-\frac{3 \sqrt{1+s^{2}}}{2}\right)+\left(\frac{1}{2}-s^{2}\right) \operatorname{arcsinh}(s)$

where the exact solution is $f(t)=\sqrt{1+t^{2}}$, and $E_{N}$ is evaluated in $[0,1]$.

Example 2. In this example we solve Eq.(1) with

$k(s, t)=s \sin (t+s)-t \cos (t-s)$

$g(s)=\frac{1}{4}\left(\left(5+s^{2}+2 s \cos (2 s)\right) \sin (s)-\left(2 s^{2}+s\right) \cos (s)\right)$ where

the exact solution is $f(t)=\sin (t)$, and $E_{N}$ is evaluated in $\left[0, \frac{\pi}{2}\right]$.
Table 1. Numerical Results for Examples

\begin{tabular}{|c|c|c|}
\hline $\mathbf{N}$ & $\boldsymbol{E}_{N}$ for Ex.1 & $\boldsymbol{E}_{\boldsymbol{N}}$ for $\mathbf{E x . 2}$ \\
\hline \hline 3 & $3.8315 \times 10^{-3}$ & $5.6884 \times 10^{-2}$ \\
\hline 5 & $8.8190 \times 10^{-7}$ & $5.1365 \times 10^{-6}$ \\
\hline 7 & $7.8724 \times 10^{-11}$ & $1.7283 \times 10^{-12}$ \\
\hline 9 & $3.2124 \times 10^{-11}$ & $1.2213 \times 10^{-15}$ \\
\hline
\end{tabular}

\section{REFERENCES}

[1] M H. Reihani, Z. Abadi, "Rationalized Haar functions method for solving Fredholm and Volterra integral equations", J. Comput. Appl. Math., vol. 200, Issue 1, pp. 12-201 March 2007.

[2] M. Rabbani, K. Maleknejad, N. Aghazadeh, "Numerical computational solution of the Volterra integral equations system of the second kind by using an expansion method", Appl. Math. Comput., Vol. 187, Issue 2, pp. 1143-1146, 15 April 2007.

[3] J. Saberi-Nadjafi, M. Heidari, "A quadrature method with variable step for solving linear Volterra integral equations of the second kind", Appl. Math. Comput., Vol. 188, Issue 1, pp. 549-554, 1 May 2007.

[4] M. Ghasemi, M. Tavassoli Kajani, E. Babolian "Numerical solutions of the nonlinear Volterra-Fredholm integral equations by using homotopy perturbation method Applied", Math. Comput., Vol. 188, Issue 1, pp. 446-449 1 May 2007.

[5] J. Biazar, H. Ghazvini, "He's homotopy perturbation method for solving systems of Volterra integral equations of the second kind", Chaos,Solitons Fractals, In Press, Available online 10 May 2007.

[6] F. Stenger, Numerical Methods Based on Sinc and Analytic Functions, Springer, New York, 1993.

[7] K E. Atkinson, The Numerical Solution of Integral Equations of the Second Kind, Cambridge University Press, 1997.

[8] R. Kress, Numerical Integral Equation, Springer-Verlag, New York, 1998.

[9] G. Evans, Practical Numerical Integration, John Wiley and Sons, 1993. 